\section{Bei COPD mit LAMA beginnen}

Suissa $S$ et al. Comparative effectiveness of LABA-ICS versus LAMA as initial treatment in COPD targeted by blood eosinophils: a population-based cohort study. Lancet Respir Med 2018; 6: $855-862$

Entgegen der GOLD-Empfehlungen erhalten Patienten mit einer chronisch obstruktiven Lungenerkrankung (COPD) häufig eine Kombinationstherapie mit inhalativen $\beta 2-\mathrm{Mi}-$ metika und Kortikosteroiden (LABAICS). Die Studie belegte die Nichtunterlegenheit von langwirksamen Anticholinergika (LAMA). Aber es gab Ausnahmen: Erkrankte mit einer Eosinophilie $>4 \%$ exazerbierten mit LABA-ICS seltener.

Die Leitlinien empfehlen LABA-ICS für Patienten mit schweren COPD-Symptomen und häufigen Exazerbationen. Der Nettogewinn im Vergleich zu LAMA sei aber unklar bei gleichzeitigen Bedenken wegen eines erhöhten Pneumonierisikos durch inhalative Kortikosteroide. In der populationsbasierten Kohortenstudie erhielten 18500 Patienten LABA-ICS und 13870 LAMA als Einstiegsbehandlung. Die Autoren verglichen die Exazerbationsraten und Pneumoniehäufigkeit der Gruppen. Dann erfolgte eine Stratifizierung nach der Eosinophilie als Biomarker für die Wirksamkeit von ICS.

Die Daten stammten aus dem britischen Clinical Practice Research Datalink, das Informationen zur medizinischen Grundversorgung von > 10 Millionen Menschen enthält. Patienten mit der Diagnose COPD und einem Alter > 55 Jahre wurden bis zu 1 Jahr beobachtet. Eine moderate Exazerbation war über die ambulante Verordnung von Prednisolon und eine schwere Exazerbation über eine Krankenhauseinweisung definiert. Nur stationär behandelte Pneumonien waren ein weiterer Endpunkt.

Nach dem Matching bildeten 12366 Erkrankte die LABA-ICS-Kohorte und 12366 die LAMA-Gruppe. Patienten mit LABA-ICS hatten öfter eine frühere Asthma-Diagnose $(43,7 \%$ vs. $33,2 \%)$ und vo- rangegangene Monotherapie mit ICS. $67 \%$ aller Personen wiesen eine Eosinophilie $>2 \%$ auf. Eine ausgeprägte Eosinophilie $>4 \%$ bestand allerdings bei nur $25 \%$. Im 1. Behandlungsjahr mit LABAICS oder LAMA unterschied sich die Häufigkeit von Exazerbationen insgesamt nicht signifikant. Die Stratifizierung nach der Eosinophilie ergab aber Unterschiede zugunsten der LABA-ICS:

- <2\%: HR 1,03 (95\%-KI 0,93-1,13),

- 2-4\%: HR 1,00 (95\%-KI 0,91-1,10),

- >4\%: HR 0,79 (0,70-0,88).

Hinsichtlich der Exazerbationsrate profitierten somit nur Patienten mit einer deutlichen Eosinophilie von der Kombination mit inhalativen Kortikosteroiden. Für die absoluten Eosinophilenwerte ergaben sich vergleichbare Resultate.

Stationäre Aufnahmen wegen einer Pneumonie kamen in der LABA-ICSGruppe häufiger vor (HR 1,37; 95\%-KI 1,17-1,60). Dies galt insbesondere, wenn Fluticason verwendet wurde (HR 1,43; 95\%-KI 1,18-1,73). Die Risikosteigerung war in den Eosinophilie-Subgruppen vergleichbar. $\geq 2$ Exazerbationen kamen bei 2766 Patienten (11,2\%) vor und traten in der LABA-ICS-Kohorte seltener auf (HR 0,87; 95\%-KI 0,79-0,97). Die Autoren führen dies auf den Anteil der Patienten mit einer Eosinophilie $>4 \%$ zurück (HR 0,78; 95\%-KI 0,63-0,97).

FAZIT

In der Beobachtungsstudie waren LABA-ICS nur bei einer deutlichen Eosinophilie und möglicherweise bei Patienten mit häufigen Exazerbationen überlegen. Dem stand eine Risikosteigerung für schwere Pneumonien gegenüber. Wenn die Eosinophilie $\leq 4 \%$ bzw. $\leq 300 / \mu$ l betrage, empfehlen die Autoren einen Behandlungsstart mit LAMA.

Dr. med. Susanne Krome, Melle 